\title{
Changing Plasma and Urinary Organic Acid Levels in a Patient with Isovaleric Acidemia during an Attack
}

\author{
YOSUKE SHIGEMATSU. ${ }^{(2)}$ MASAKATSU SUDO, TORU MOMOI, YASUHIRO INOUE, \\ YASUHIRO SUZUKI, AND JUNJI KAMEYAMA
}

Department of Pediatrics, Faculty of Medicine, Kyoto University, Sakyo-ku, Kyoto, Japan [Y.S., T.M., Y.I.J; Department of Pediatrics, Fukui Medical School, Fukui, Japan [M.S.]; Chest Disease Research Institute, Kyoto University, Kyoto, Japan [Y. Suzuki]; and Department of Pediatrics, Uji-Tokushukai Hospital, Uji, Japan [J.K.]

\begin{abstract}
Summary
Organic acids in plasma and urine of a patient with isovaleric acidemia were measured serially during a severe ketoacidotic attack. Urinary ketone bodies, lactic acid and 2-hydroxy- $n$-butyric acid changed in parallel with the plasma isovaleric acid concentration, which was correlated closely with the severity of the clinical symptoms. A 2-day lag was observed between the time of the peak plasma isovaleric acid level and that of the highest urinary excretion of 3-hydroxyisovaleric acid, methylsuccinic acid, mesaconic acid and 2-hydroxyisobutyric acid, the total quantity of which amounted to one-third of that of isovalerylglycine on the 4th day of the attack. Urinary isovalerylglycine excretion remained at a plateau for $\mathbf{4}$ days after the highest level of plasma isovaleric acid was observed. The decrease of urinary hippuric acid excretion during the attack continued until the patient had recovered enough to be able to eat. Benzoic acid administration concomitant with leucine and glycine canceled the effect of glycine in lessening the rise of plasma isovaleric acid concentration after a leucine load.
\end{abstract}

\section{Speculation}

Whereas the glycine-conjugating pathway plays a major role in clearing accumulated isovaleryl-CoA and isovaleric acid in isovaleric acidemia, $\omega$ - and $(\omega-1)$-oxidation systems may also make a considerable contribution to detoxifying isovaleric acid, because their metabolites are excreted in large quantities in the urine. The present study shows, however, that these oxidaton systems cannot function effectively in the early stage of a severe attack and that the capacity of the glycine-conjugating pathway is not sufficient, so other methods of removing accumulated isovaleric acid in body fluids, such as blood exchange transfusion, may be needed.

Isovaleric acidemia is an inherited abnormality of leucine degradation caused by a deficiency of isovaleryl-CoA dehydrogenase. More than 30 patients have been reported since the first description by Tanaka et al. (15). The patients showed a wide spectrum of clinical findings from the acute neonatal form to the chronic intermittent form, which may be related to the severity of dehydrogenase deficiency. The metabolic alteration consists mainly of glycine-conjugation that forms isovalerylglycine from isovalerylCoA (16). In addition to isovalerylglycine, which is a characteristic metabolite excreted in the urine of these patients, other organic acids such as 3-hydroxyisovaleric acid and methylsuccinic acid have been noted in the urine (17). However, Duran et al. (6) recently reported that neither 3-hydroxyisovaleric acid nor methylsuccinic acid could be found in the urine of patients with the chronic intermittent type and normal development. We were able to observe a Japanese patient and to investigate organic acids in the plasma and urine during a severe ketoacidotic attack. The interesting changes in the organic acids of this patient are reported here.

\section{CASE REPORT}

A Japanese girl, M.U., was born without asphyxia after an uneventful pregnancy of 40 weeks on June 20, 1975; birth weight was $3530 \mathrm{~g}$. She was the first child of healthy parents. It was not a consanguineous marriage. A younger sister is healthy.

During the first year after birth, she nursed well and gained weight smoothly with normal developmental milestones. After weaning, she did not eat meat, eggs or cow's milk willingly, but did eat rice and vegetables. She experienced her first ketoacidotic attack during the course of bronchopneumonia at $1 \frac{1}{2}$ years of age. Drowsiness followed by frequent vomiting persisted for about 1 week, and foul breath and marked leucocytopenia were noted.

Since then she has had four moderate to severe attacks, which usually followed common cold symptoms. She first visited our hospital because of vomiting at the age of 4 years and 10 months. Loss of hair was prominent, and a large amount of isovalerylglycine in the urine was detected by gas chromatography/mass spectrometry. Other abnormal laboratory data on admission were: white blood cell count, $2,100 / \mathrm{cmm}$; blood $\mathrm{NH}_{3,}, 123 \mu \mathrm{g} / \mathrm{dl}$ (normal range: $20-60)$; serum uric acid, $18.5 \mathrm{mg} / \mathrm{dl}$ (2.3-8.0); blood $\mathrm{pH}$, 7.35; base excess, $-9.5 \mathrm{mmole} / \mathrm{liter}$ and strongly positive urinary acetone. Plasma and urinary amino acids were within the normal range. After recovery from drowsiness she had no motor disability and her intelligence was normal (IQ, 102). EEG and computerized tomography of the head showed no abnormalities.

A diagnosis of isovaleric acidemia was made. She was given oral glycine, 3-6 g/day, when she complained of discomfort and the urinary acetone was positive. There were several such episodes during the following four months, but no vomiting attack.

At the age of 5 years and 3 months, she was readmitted to our hospital for detailed metabolic studies. Informed consent was obtained from her parents. Early on the second hospital day, she coughed slightly, but the urinary acetone was negative. Oral glycine was given to investigate its effect on plasma isovaleric acid; however, she soon complained of feeling ill, and $5 \mathrm{~h}$ after the glycine load she started to vomit frequently. The urinary acetone became positive. In spite of fluid supplement including glucose with insulin and rectal glycine administration $(200 \mathrm{mg} / \mathrm{kg}$ body weight) (12), she became lethargic. Rectal glycine was continued as long as possible despite increasing diarrhea. Her clinical symptoms during the attack are illustrated in Figure 3.

\section{MATERIALS AND METHODS}

Blood specimens were obtained at 9 a.m. and at 6 p.m. on the 2nd and 3rd days of the attack, and at 9 a.m. on the following 
days. The collection of a 24 -h urine specimen was started at 8 a.m. Urine and plasma samples were kept at $-20^{\circ} \mathrm{C}$ before the analysis of organic acids.

The standard substances used here were obtained from Nakarai Chemicals Ltd., Kyoto, Japan, except for isovalerylglycine which was synthesized by the method of Bondi and Eissler (4) with glycine and isovalerylchloride. N,O-bis(trimethylsilyl)-trifluoroacetamide was purchased from Pierce Chemical Co., Rockford, IL., U.S.A. Silicone OV-17 (3\%) on Chromosorb W (AW-DMCS) (80100 mesh) and neopentylglycoladipate $(5 \%)+\mathrm{H}_{3} \mathrm{PO}_{4}(2 \%)$ on Chromosorb W (AW-DMCS) (100-120 mesh) were obtained from Wako Pure Chemical Industries Ltd., Osaka, Japan.

Urinary organic acids were extracted from 24-h urine samples by the procedure described elsewhere (14). The extracted acids were trimethylsilylated with $200 \mu \mathrm{l}$ of $N, \mathrm{O}$-bis(trimethylsilyl)-trifluoroacetamide at $60^{\circ} \mathrm{C}$ for $2 \mathrm{~h}$. Every sample was analysed within a few $\mathrm{h}$ after silylation with a gas chromatograph, JGC20K (Japan Electron Optics Laboratory Ltd., Tokyo, Japan) equipped with a data analysing microcomputer, C-R IA CHROMATOPAC (Shimadzu Seisakusho Ltd., Kyoto, Japan), and a gas chromatograph/mass spectrometer, JMS D300 double focusing mass spectrometer, with a computer system, JMA2000 (Japan Electron Optics Laboratory Ltd., Tokyo, Japan). The injection port and the glass sample line, including the glass jet separator, between the gas chromatograph and the mass spectrometer were at $250^{\circ} \mathrm{C}$, and the ion source temperature was $150^{\circ} \mathrm{C}$. Ionizing energy was $70 \mathrm{eV}$ and accelerating voltage was $3 \mathrm{KV}$. The column was a $2 \mathrm{~m} \times 3 \mathrm{~mm}$ glass coil packed with $3 \% \mathrm{OV}-17$ on chromosorb W (AW-DMCS), and its temperature was programmed at $5^{\circ} \mathrm{C}$ / min from $80-280^{\circ} \mathrm{C}$. The carrier gas was helium with a flow rate $40 \mathrm{ml} / \mathrm{min}$.

Quantitative determinations of urinary organic acids were carried out with computer-assisted selected ion monitoring except for isovalerylglycine and hippuric acid, the values of which were calculated from the peak areas on the gas chromatogram. The monitored ion-fragments, specific for the respective silylated acids, were: lactic acid $191 \mathrm{~m} / \mathrm{z}, 2$-hydroxyisobutyric acid $233 \mathrm{~m} / \mathrm{z}, 2$ hydroxy- $n$-butyric acid $233 \mathrm{~m} / \mathrm{z}, 3$-hydroxy- $n$-butyric acid $191 \mathrm{~m} /$ z, 3-hydroxyisovaleric acid $247 \mathrm{~m} / \mathrm{z}$, acetoacetic acid $231 \mathrm{~m} / \mathrm{z}$, methylsuccinic acid $261 \mathrm{~m} / \mathrm{z}$, mesaconic acid $259 \mathrm{~m} / \mathrm{z}$, and heptadecanoic acid (internal standard) $327 \mathrm{~m} / \mathrm{z}$. The peak areas on the mass chromatogram were measured in traces by a digital analyser, Model NOP-AMO3 (KONTRON MESSGERÄTE GMBH, Eching, West Germany). The values obtained were corrected for the recovery of organic acids.

Plasma isovaleric acid was determined by the following method: a mixture of $1.0 \mathrm{ml}$ of plasma, $60 \mu \mathrm{g}$ of $n$-valeric acid as an internal standard and $20 \mathrm{ml}$ of chloroform-methanol (v/v 2:1) was filtered and the upper layer, obtained after mixing with $4 \mathrm{ml}$ of $0.1 \mathrm{~N}$ $\mathrm{NaOH}$, was concentrated. After $4 \mathrm{ml}$ of saturated $\mathrm{NaCl}$ solution was added, it was acidified to $\mathrm{pH} 1.0$ with $6 \mathrm{~N} \mathrm{HCl}$ and extracted twice with ethylether, which was evaporated to dryness after 0.2 $\mathrm{N}$ methanolic $\mathrm{NaOH}$ was added. Two tenths $\mathrm{ml}$ of $6 \mathrm{~N} \mathrm{HCl}$ and then $0.5 \mathrm{ml}$ of ethylether were added to the residue, and the mixture was dehydrated with anhydrous $\mathrm{Na}_{2} \mathrm{SO}_{4}$. These extraction procedures were carried out at $4^{\circ} \mathrm{C}$. The ether solution was injected into a JGC-2OK gas chromatograph with a $2 \mathrm{~m} \times 3 \mathrm{~mm}$ glass coil column packed with $5 \%$ neopentylglycoladipate $+2 \%$ $\mathrm{H}_{3} \mathrm{PO}_{4}$ on Chromosorb W (AW-DMCS), the temperature of which was programmed at $2^{\circ} \mathrm{C}$ from $80-140^{\circ} \mathrm{C}$. Correction for recovery was done.

\section{RESULTS}

Figure 1 shows the gas chromatograms of silylated organic acids in the urine of the patient when in a stable condition $(A)$, and in

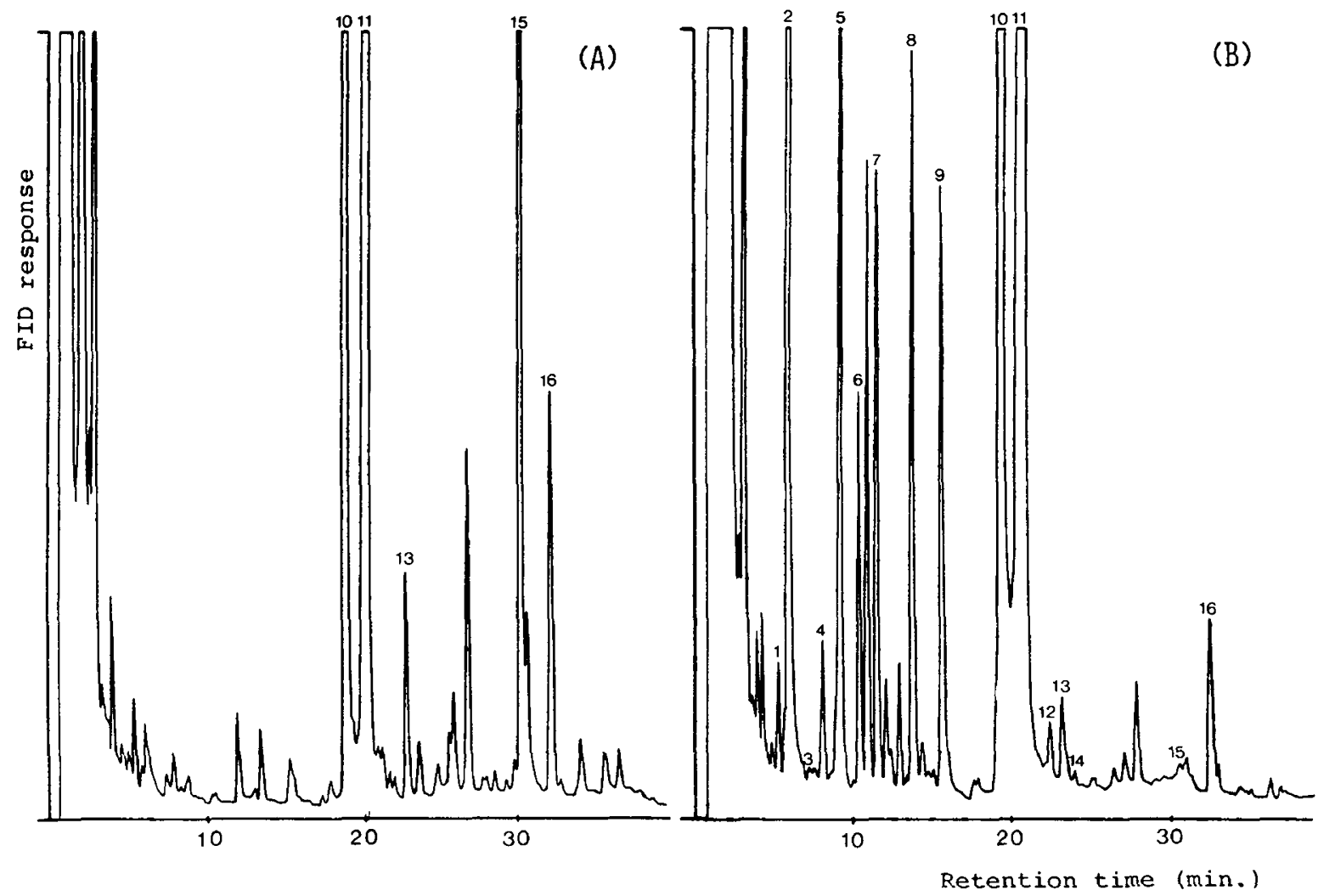

Fig. 1. Gas chromatograms of silylated organic acids in patient's urine. $(A)$ in stable condition, $(B)$ during convalescence after attack. Peak identifications are: (1) 2-hydroxyisobutyric acid; (2) lactic acid; (3) 2-hydroxy- $n$-butyric acid; (4) 3-hydroxy- $n$-butyric acid + 3-hydroxyisobutyric acid; (5) 3-hydroxyisovaleric acid; $(6,7)$ acetoacetic acid; (8) methylsuccinic acid + succinic acid + fumaric acid; (9) mesaconic acid; (10) isovalerylglycine (di-TMS); (11) isovalerylglycine (mono-TMS); (12) 4-hydroxybenzoic acid; (13) 4-hydroxyphenylacetic acid; (14) octanedioic acid; (15) hippuric acid; and (16) heptadecanoic acid (internal standard). 


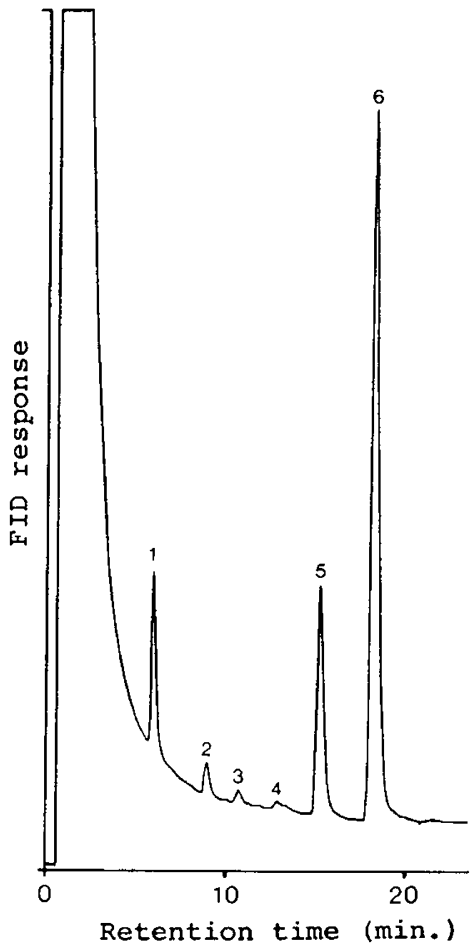

Fig. 2. Gas chromatogram of plasma volatile organic acids of the patient. Peak identifications are: (1) acetic acid: (2) propionic acid; (3) isobutyric acid; (4) $n$-butyric acid: (5) isovaleric acid; and (6) $n$-valeric acid (internal standard).

the convalescent stage following an attack $(B)$. Although isovalerylglycine was the only characteristic metabolite in the stable condition, many other acids, shown in Figure 1, were found during the attack, but only small quantities of medium-chain dicarboxylic acids were detected.

Figure 2 shows the gas chromatogram of volatile organic acids in the patient's plasma when she started to vomit. Plasma isovaleric acid concentrations of healthy adult and children were below $0.6 \mu \mathrm{g} / \mathrm{ml}(n=8)$ and those of the patient when in a stable condition ranged from $4-8 \mu \mathrm{g} / \mathrm{ml}$.

Figure 3 shows the changing plasma isovaleric acid and urinary organic acids during the attack in relation to clinical condition and therapy. The concentration of plasma isovaleric acid before oral glycine administration on the first day of the attack was 30.8 $\mu \mathrm{g} / \mathrm{ml}$, and it decreased transiently to $6.8 \mu \mathrm{g} / \mathrm{ml} \mathrm{l} \mathrm{h} \mathrm{later,} \mathrm{then}$ increased progressively to reach $813 \mu \mathrm{g} / \mathrm{ml}$ on the evening of the 2 nd day of attack. Large quantitites of ketone bodies (3-hydroxy$n$-butyric acid and acetoacetic acid), lactic acid and 2-hydroxy- $n$ butyric acid were excreted in the urine on the 2 nd day of the attack, when 3-hydroxyisovaleric acid, methylsuccinic acid, mesaconic acid, and 2-hydroxyisobutyric acid were found in small amounts. The latter four metabolites reached their highest values on the 4th day of the attack, when the former acids were decreasing. Urinary isovalerylglycine excretion was as high as $1.45 \mathrm{~g} /$ day on the 3rd day of the attack, remained high for 4 days, and returned to the basal level on the 13th hospital day. Urinary hippuric acid, which decreased during the attack, increased as the patient recovered and was able to eat. The severity of her clinical condition correlated most closely to the plasma isovaleric acid concentration and urinary excretion of ketone bodies, lactic acid and 2-hydroxy- $n$-butyric acid.

Figure 4 shows the results of the leucine loading test. The dose of leucine was $25 \mathrm{mg} / \mathrm{kg}$, as advocated by Yudkoff et al. (21) because it was thought that the patient could not tolerate $125 \mathrm{mg} /$ $\mathrm{kg}(12)$. Loading tests were carried out on the 13th, 15th and 17th hospital days; leucine with $250 \mathrm{mg} / \mathrm{kg}$ of glycine, leucine without glycine and leucine with $250 \mathrm{mg} / \mathrm{kg}$ of glycine and $200 \mathrm{mg}$ of benzoic acid were given. Before each loading test, the patient was clinically well, and the plasma isovaleric acid concentration ranged between $4.8-7.2 \mu \mathrm{g} / \mathrm{ml}$. None of these loading tests provoked a vomiting attack. The rise of plasma isovaleric acid after leucine loading with glycine was less than after leucine without glycine, whereas the rise after leucine, glycine and benzoic acid was almost the same as the rise after leucine alone.

\section{DISCUSSION}

The main metabolic pathway of accumulated isovaleryl-CoA in isovaleric acidemia is thought to be the formation of isovalerylglycine by glycine conjugation (16). It is known that other products from isovaleryl-CoA are 3-hydroxyisovaleric acid in the ( $\omega-1)$ hydroxylation system and methylsuccinic and mesaconic acids in the $\omega$-oxidation system. These acids in addition to isovalerylglycine have been found in the urine during episodes of ketoacidosis in this disease $(2,5,17,18)$; however, the changes of plasma and urinary organic acids during attacks of isovaleric acidemia have not been studied in detail.

The features which were noticed in the changing organic acid levels during the attack in the present study were as follows: (1) urinary ketone bodies, lactic acid and 2-hydroxy- $n$-butyric acid paralleled plasma isovaleric acid levels which were correlated closely with the severity of the clinical symptoms; (2) a 2-day lag was observed between the time of the plasma isovaleric acid peak and the maximum urinary excretion of 3-hydroxyisovaleric acid, mesaconic acid and methylsuccinic acid: (3) urinary isovalerylglycine excretion remained at a plateau for 4 days after the plasma isovaleric acid peak; and (4) urinary hippuric acid excretion was low when the patient could not eat at all.

The formation of ketone bodies, lactic acid and 2-hydroxy- $n$ butyric acid was thought to be induced by accumulated isovaleric acid, which could disturb the energy metabolism by inhibiting oxidative phosphorylation $(10,11)$. The present data show that the level of urinary ketone bodies reflects that of accumulated isovaleric acid in the body fluids. Therefore, it is clinically useful to measure urinary ketone bodies in order to judge the condition of a patient with isovaleric acidemia. The parents are instructed to give the patient glycine when the urinary ketone test is positive.

Serial examinations of urinary 3-hydroxyisovaleric, methylsuccinic, mesaconic and 2-hydroxyisobutyric acids showed that the amounts of these acids varied greatly during the attack; maximum excretion occurred during the recovery stage (the 4th day of the attack). This finding shows that the pattern of urinary organic acids in isovaleric acidemia varies with the stage of the attack; this may explain the discrepancies in the data on urinary organic acids in this disease as reported by several authors (6).

The two-day lag between the time of the maximum plasma isovaleric acid level and that of the maximum excretion of urinary 3-hydroxyisovaleric and other acids is very interesting because it indicates that the $\omega$ - and $(\omega-1)$-hydroxylation systems do not function effectively during the severe phase of the attack in spite of the presence of a large amount of their substrate. The $\omega$ hydroxylation system has been reported to be located in the cytoplasm of liver cells or other cells and to require NADPH, $\mathrm{O}_{2}$ and cytochrome $P_{450}(13,19)$, and the $(\omega-1)$-hydroxylation system is thought to be similar to the $\omega$-hydroxylation system, although there are several differences between them $(3,9,14)$. It is known that NADPH is generated by cytoplasmic dehydrogenases and by mitochondrial transhydrogenation from $\mathrm{NADH}$ and that the transhydrogenation requires energy such as ATP (20). So it is very possible that in critically ill patients the disturbance in energy metabolism relating to the generation of NADPH is responsible for the inhibition of the $\omega$ - and $(\omega-1)$-hydroxylation systems. The physiologic significance of the $\omega$ - and ( $\omega-1)$-hydroxylation systems has been noted in "non-ketotic dicarboxylic aciduria" (8), in which it was thought that defective $\beta$-oxidation is primary and that accumulated short- or medium-chain acyl-CoA is metabolized by these oxidation systems. In isovaleric acidemia, $(\omega-1)$ hydroxylation may be more available during attacks than $\omega$ - 


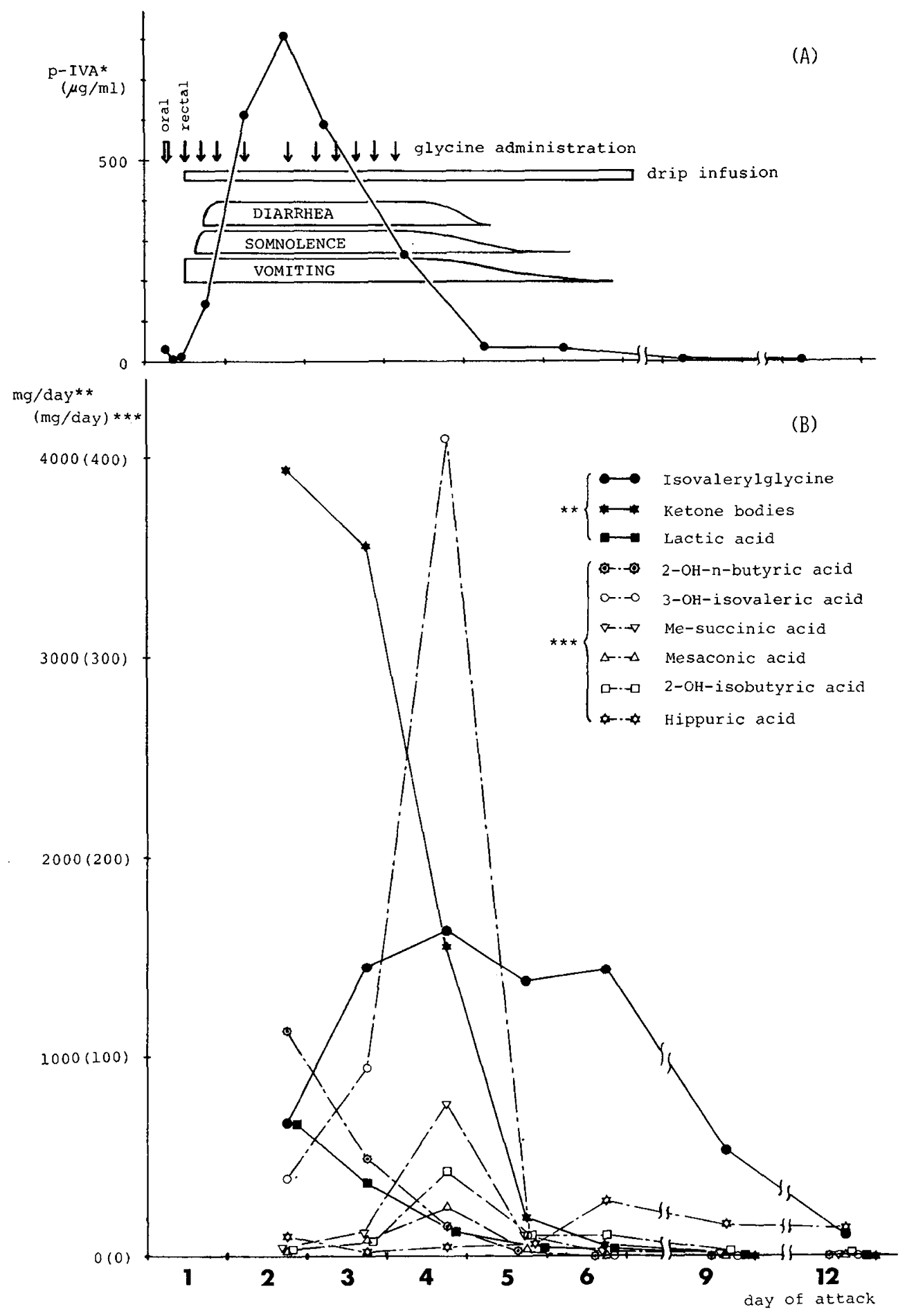

Fig. 3. Changes of plasma isovaleric acid $(A)$ and urinary organic acids $(B) .{ }^{*}$ plasma isovaleric acid.

oxidation, to which short-chain fatty acids may possess relatively poor susceptibility (14). The products of these oxidation systems, 3-hydroxyisovaleric, methylsuccinic and mesaconic acids, as well as isovalerylglycine have a higher renal clearance than free isovaleric acid, which has been reported to be almost absent in the urine (12). The quantities of these three acids in the urine on the 4th day of the attack were one-third the concentration of isovalerylglycine. Urinary 2-hydroxyisobutyric acid levels changed in parallel with 3-hydroxyisovaleric acid levels. Goodman et al. (7) found that 2-hydroxyisobutyric acid was excreted abundantly in the urine of a patient with "multiple acyl-CoA dehydrogenase deficiency" and speculated that it was formed by the ( $\omega-1)$-hydroxylation of isobutyryl-CoA derived from valine. It is, however, not clear whether the 2-hydroxyisobutyric acid in the urine of our patient was derived from valine or not, because the dehydrogenase which acts on isobutyryl-CoA seems to be intact in isovaleric acidemia.

It is thought that such an inhibition of energy metabolism as that mentioned above could influence the glycine conjugation of isovaleric acid which requires ATP; however, this may not be the only cause of the third feature in the present study. On the basis of their experience in treating neonatal isovaleric acidemia with glycine, Cohn et al. (5) stated that increased urinary isovalerylglycine excretion followed glycine administration and that many days were needed for fat-soluble isovaleric acid to be cleared from tissue sites. 


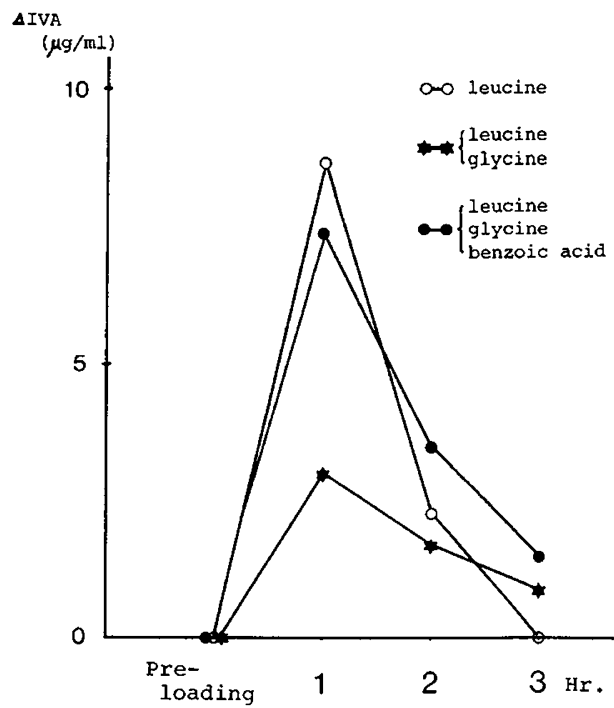

Fig. 4. Plasma isovaleric acid after oral leucine load without glycine, with glycine, and with glycine and benzoic acid.

The course of urinary isovalerylglycine excretion in our patient suggests that the capacity of the glycine conjugation pathway can rise only slowly during an attack and that there is a limit to that capacity irrespective of the amount of isovaleric acid and the supply of glycine. Glycine administration may not always be sufficient to prevent severe attacks even though it makes a considerable contribution to the enhancement of glycine conjugation. Nevertheless, glycine conjugation appeared to play a major role in clearing accumulated isovaleric acid, because isovalerylglycine comprised three-fourths of the metabolites of isovaleric acid on the fourth day of the attack.

In discussing the capacity of the glycine conjugating pathway, substances competing for the conjugation such as benzoic acid and salicylic acid, have been mentioned $(1,12)$. The explanation of benzoic acid's harmful effect was based on the observation that urinary hippuric acid excretion decreased during ketoacidotic attacks in isovaleric acidemia; however, one must remember that the patient can not take fruits and other foods containing benzoic acid during an attack. In the present study, indeed, urinary hippuric acid excretion increased when the patient had recovered enough to drink liquids such as fruit juice. The unfavorable effect of benzoic acid was clearly shown by the loading test. The administration of benzoic acid with leucine and glycine caused the same increase of plasma isovaleric acid as the administration of leucine without glycine.

The loading test showed the significant effect of glycine administration in reducing the plasma level of isovaleric acid. However, it is also true that the oral and rectal administration of glycine could not prevent the severe ketoacidotic attack of our patient. A more effective treatment may be the combined administration of glycine and a leucine-poor amino acid mixture.

The present study shows clearly that the restriction of compounds competing with isovaleric acid for glycine conjugation increase the effectiveness of glycine. Furthermore, we think that the intake of competitive substances in $\omega$ - and ( $\omega$-1)-oxidation, which include fatty acids especially medium-chain fatty acids (14) should be avoided. In view of these considerations, further detailed biochemical studies of $\omega$ - and ( $\omega-1)$-oxidation should be undertaken.

\section{REFERENCES AND NOTES}

1. Ando, T.. Klingberg. W. G., Ward, A. N., Rasmussen, K.. and Nyhan, W. L.: Isovaleric acidemia presenting with altered metabolism of glycine. Pediatr. Res. 5. 478 (1971)

2. Ando, T.. Nyhan, W. L., Bachmann, C., Rasmussen. K., Scott, R., and Smith, E. K.: Isovaleric acidemia: identification of isovalerate, isovalerylglycine, and 3 hydroxyisovalerate in the urine of a patient previously reported as having butyric and hexanoic acidemia. Pediatrics, 82: 243 (1973).

3. Björkhem, I. and Danielsson. H.: $\omega$ - and $(\omega-1)$-oxidation of fatty acids by rat liver microsomes. Eur. J. Biochem., 17: 450 (1970).

4. Bondi. S. and Eissler, F.: Über lipoproteide und die deutung der degenerativen zellverfettung. Biochem. Zeitschrift. 23: 499 (1910).

5. Cohn. R. M.. Yudkoff. M., Rothman. R., and Segal, S.: Isovaleric acidemia: use of glycine therapy in neonates. N. Engl. J. Med., 299: 996 (1978).

6. Duran, M.. van Sprang, F. J., Drewes, J. G., Bruinvis, L., Ketting, D., and Wadman. S. K.: Two sisters with isovaleric acidemia. multiple attacks of ketoacidosis and normal development. Eur. J. Pediatr., 1.31: 205 (1979).

7. Goodman, S. I., McCabe, E. R. B., Fennessey, P. V.. and Mace, J. W.: Multiple acyl-CoA dehydrogenase deficiency (glutaric aciduria type II) with transien hypersarcosinemia and sarcosinuria: possible inherited deficiency of an electron transfer flavoprotein. Pediatr. Res, 14: 12 (1980)

8. Gregersen. N., Rosleff. F.. Kølvraa. S., Hobolth. N., Rasmussen, K., and Lauritzen, R.: Non-ketotic $\mathrm{C}_{1 ;}-\mathrm{C}_{10}$-dicarboxylic aciduria: biochemical investigations of two cases. Clin. Chim. Acta, 102: 179 (1980)

9. Heinz. E., Tulloch, A. P. and Spencer, J. F. T.: Hydroxylation of oleic acid by cell-free extracts of a species of Torulopsis. Biochim. Biophys. Acta, 202: 49 (1970).

10. Hird, F. J. R. and Weideman, M. J.: Oxidative phosphorylation accompanying oxidation of short chain fatty acids by rat liver mitochondria. Biochem. J.. 98: 378 (1966).

11. Kamerling. J. P.. Gerwig. G. J., Duran, M.. Ketting, D., and Wadman, S. K. The absolute configuration of urinary 2-hydroxybutyric acid in patients with ketosis and lactic acidosis. Clin. Chim. Acta, 88: 183 (1978).

12. Krieger, 1. and Tanaka, K.: Therapeutic effects of glycine in isovaleric acidemia Pediatr. Res., /0: 25 (1976).

13. Lu. A. Y. H.. Junk, K. M.. and Coon, M. J.: Resolution of the cytochrome P-450containing $\omega$-hydroxylation system of liver microsomes into three components. J. Biol. Chem.. 244: 3741 (1969)

14. Shigematsu. Y.. Momoi. T., Sudo, M., and Suzuki, Y.: ( $\omega-1)$-Hydroxymonocarboxylic acids in the urine of infants fed medium-chain triglycerides. Clin Chem. 27: 1661 (1981).

15. Tanaka. K., Budd, M. A., Efron, M. L., and Isselbacher, K. J.: Isovaleric acidemia: a new genetic defect of leucine metabolism. Proc. Natl. Acad. Sci. 56: $236(1966)$

16. Tanaka, K. and Isselbacher. K. J.: The isolation and identification of N-isovalerylglycine from urine of patients with isovaleric acidemia. J. Biol. Chem., 242 2966 (1967).

17. Tanaka. K., Orr, J. C.. and Isselbacher. K. J.: Identification of 3-hydroxyisovaleric acid in the urine of a patient with isovaleric acidemia. Biochim. Biophys. Acta, 152: 638 (1968)

18. Truscott. R. J. W.. Malegan. D., McCairns. E., Burke, D., Hick, L., Sims, P. Halpern, B.. Tanaka, K., Sweetman, L., Nyhan. W. L., Hammond, J., Bumack, C., Haan, E. A.. and Danks, D. M.: New metabolites in isovaleric acidemia. Clin. Chim. Acta, 110: 187 (1981).

19. White. A.. Handler, P., Smith, E. L., Hill, R. L.. and Lehman, I. R.: Lipid Metabolism I. Principles of biochemistry. pp 577-584 (1978) McGRAW-HILL KOGAKUSHA Tokyo.

20. White, A., Handler, P.. Smith, E. L., Hill, R. L., and Lehman, I. R.: Biological Oxidation I \& II. Principles of biochemistry. pp 323-422 (1978) McGRAWHILL KOGAKUSHA Tokyo.

21. Yudkoff. M.. Cohn. R. M., Puschak. R.. Rothman. R.. and Segal. S.: Glycine therapy in isovaleric acidemia. J. Pediatr., 92: 813 (1978).

22. Requests for reprints should be addressed to: Dr. Yosuke Shigematsu, Department of Pediatrics. Faculty of Medicine. Kyoto University, Sakyo- ku, Kyoto, 606. Japan.

23. Received for publication September 4, 1981 .

24. Accepted for publication March 4, 1982 